\title{
Research on the Influence of the Internet on Teenagers' Psychology and Behavior
}

\author{
Nuoya $\operatorname{Lin}^{1}$ \\ ${ }^{1}$ Shandong Normal University, Jinan, Shandong 250000, China \\ Email:743873372@qq.com
}

\begin{abstract}
With the development of the Internet, people have gradually entered the information society. As the internet brought technological development, it also has a profound impact on the psychological behavior of young people. This article uses socialization, use and satisfaction theories and cognitive theories, psychological and behavioral causes and characteristics to explore the pros and cons of the Internet: it has a positive effect on teenagers' learning and social interactions, on the contrary, the Internet has a negative effect on the mind and body of teenagers. Then put forward countermeasures from the perspectives of national laws, school parents and young people themselves which is conducive to strengthening the governance of young people's network and helping young people to surf the Internet healthily.
\end{abstract}

Keywords: Internet, teenager, psychology and behavior, governance of youth Internet use

\section{BACKGROUND}

With the development of the Internet, people have gradually entered the information society. While the Internet has brought technological development, it has also greatly changed people's lives, and brought a lot of convenience to people. We can shop, study, and work without leaving home. People can make friends and watch shows online. As a group of teenagers who have grown up with the internet, its psychology and behavior are deeply influenced by the Internet.

Adolescents refer to people between the ages of 10 and 19 who are in the adolescent age group [1]. The most prominent feature of contemporary young people is that they are aboriginals who grew up with the Internet. They are full of curiosity about new things that appear on the Internet and have a strong ability to imitate, so they will make friends, study and join subcultural groups online. Meanwhile, due to lack of sufficient social experience, no education on healthy Internet access and failure to form a correct outlook on life and values, teenagers are extremely easily affected by the bad information on the Internet which will cause adolescents' ambiguity about right and wrong and a decline in their moral awareness even hurt their physical and mental health. Moreover, according to the "Research Report on the Internet Usage of National Minors in 2019" [2], the number of underage Internet users in my country was 175 million and internet penetration rate among minors reached $93.1 \%$. Among them, the proportion of underage netizens who use the Internet for learning is $89.6 \%$. However, the proportion of playing online games is as high as $61 \%$ and the rate of viewing short videos is $46.2 \%$. Meanwhile, "The 47 th Statistical Report on Internet Development in China" (2021) pointed out that as of December 2020, the proportion of netizens in middle school, high school and technical school is $60.9 \%$. Internet penetration among minors is very high and they are becoming the main force in Internet use. In the meantime, the reason for most teenagers to use the Internet is to gain a sense of identity from peers or adults. However, some information on the Internet is so complex that it is difficult to distinguish the authenticity and the use groups are complex and diverse which is difficult to control. The empathy, good and evil ideas of young people are easily incited which leads them tend to be at a loss or blindly follow the temptation of the Internet.

The Internet breaks through the limitations of time, space and location, with its characteristics of fast spreading speed and wide spreading range promoting the development of youth quality education and the improvement of their learning ability. The development of online education and distance education breaks through the limitations of place and time which enables young people to learn through the Internet. Meanwhile, 
the Internet is much more flexibility than the traditional curriculum education. It provides a new way for young people to improve their artistic appreciation and learn traditional culture. For example, teenagers can get to know The National Treasure through bilibili which is a subculture video website. They can also learn the advanced culture from other different countries, for example, watching British and American dramas has become a choice for more and more teenagers to release themselves. While teenagers use the Internet to acquire knowledge, the existence of Internet fraud, pornographic violence, feudal superstition, and Internet addiction also have a negative impact on the growth of teenagers. The main purpose of this article is using the Sociology, Communication and Education theories to comprehensively analyze and discuss the pros and cons of Internet use on the psychology and behavior of young people, and to propose solutions to existing problems.

\section{THEORETICAL BASIS}

\subsection{Socialization theory}

Socialization refers to the process in which natural persons participate in social life through contact and interactions with society, and gradually transform into social persons. Everyone can adapt to social standards of behavior after being socialized, which is an important basis for people to participate in social interactions. Socialization includes two aspects. One is the process in which the society educates individuals, and the other is the process in which members of society conduct education through interactions. The necessary conditions for socialization mainly include the personal biological basic environment and the external social environment. The external social environment includes family, school, political system, group, etc. The basic personal biological conditions are mainly reflected in the individual's language ability, learning ability, thinking ability, etc. Socialization runs through people's lives, and adolescence is one of the important stages [3].

In the era of new media, the emergence of social media has had many impacts on the socialization of young people. Therefore, many scholars use socialization to analyze the impact of the Internet on young people. For example, a short video networks such as Tik Tok play an important impact on the socialization of teenagers. The novel and comprehensive video content in the short video has a high acceptance and a strong sense of identity for young people which actively promote the formation of correct social norms and behavioral standards in youth social interactions. On the other hand, incorrect concepts such as money worship, comparison, pornography, and violence in the short video have a negative impact on the formation of the correct outlook on life and values of young people.

\subsection{Theory of Use and Satisfaction}

The theory of use and satisfaction believes that audiences use specific media based on specific psychological motives and psychological needs, and the media use of audiences is also the process by which their specific needs are met [4]. Previous scholars mostly explored the psychological needs and goals of teenagers when they used newspapers and TV broadcasts. Nowadays, in the Internet age, many scholars have begun to adapt to the age to study the mentality of young people participating in Internet activities. Qiao lei Jiang, in "The Psychology, Risks and Countermeasures of Using Short Videos for Teenagers" mentioned that through use and gratification theory teenagers use bilibili and TikTok by swiping, liking, commenting, barrage, sharing, saving, shooting, uploading, etc. The psychological satisfaction adolescents obtain by using the short video platform includes: entertainment, social interactions, information acquisition, self-exhibition, record storage, escape from reality and other psychological needs [4]. Use and satisfaction are closely linked to cognitive theory. Many conclusions only study the psychology and behavior of adolescents using the Internet without proposing specific solutions. On the other hand, the scholar $\mathrm{Yu}$ lin Zan mentioned that Internet addiction is the negative effect of teenagers using the Internet.

Internet addiction includes the addiction of Internet porn, communication, information overload, game and audiovisual and so on. Meanwhile it brings adverse effects such as obesity, tension with other family members, depression and so on [5].

\subsection{Cognitive theory}

Social cognition in social cognition theory is not equal to cognition in the general sense. Its research scope includes the individual's understanding of the external and internal characteristics of others (for example, personality, emotions, etc.) and the understanding of interpersonal relationships. Socialcognitive theory emphasizes that the construction of cognition can play an important guiding role in the development of individual behavior. In the process of studying individual behavior, it focuses on the cognition, and the role of expectations, motivations, goals and other factors. Sternberg Sewing attributed the "socialcognitive" theory to a dynamic social learning theory [6]. At the same time, under the background that young people use the Internet more and more frequently, there is a cognitive dissonance of the Internet. Among them, the most typical manifestation is Internet addiction. Although online games provide a perfect virtual platform for the boring and long-lasting study life of teenagers in real life and bring temporary satisfaction and happiness to young people. But after the online 
game behavior, facing real life, they can't find the external reason for their online game behavior, which seriously threatens the teenagers' self-evaluation of their own image. They often have ambivalence of guilt and restlessness, leading to cognitive dissonance [7].

\section{THE PSYCHOLOGICAL AND BEHAVIORAL REASONS OF TEENAGERS USING THE INTERNET}

Psychologically, most young people use the Internet for the following two reasons: One is for learning and education. The Internet has a large amount of information and is easy to retrieve, rich in content, mobility, convenience, saving time and effort. At the same time, the Internet breaks geographical and time constraints. Compared with adults, the net generation that has grown up with the Internet. With the help of proficient use and understanding of the Internet, young people are more inclined to use the Internet for learning which has become a habit for them. However, the offline method of teaching through teachers alone and transferring knowledge has not been able to satisfy the thirst for knowledge of young people. Many universities have also opened online courses and upload content-rich online courses. They can access the cutting-edge scientific and technological achievements and news information of different disciplines through the Internet. Furthermore, it can let young people understand different countries and different cultures, broaden their horizons, and satisfy young people's curiosity to explore the world.

The second is to entertain and relax. The Internet has the characteristics of youthfulness, fun, cultural diversity, and gathering. It attracts young people to participate in Internet activities and promotes the development of subcultures. It also satisfies the emotions that young people cannot get in reality. With the development of the Internet, a number of cultures that meet the preferences of young people such as online games, two-dimensional, star chasing, and social media such as bilibili, ins, and Twitter have emerged. At the same time, some young people use the Internet to satisfy their own comparisons or to show off their desire for psychological performance and self-realization. Through online games, they can gain the respect and recognition of their peers. From an emotional point of view, there are also adolescents who psychologically depressed and lack communication with others in reality, including those who lack parental care in reality or who are in a rebellious period and cannot be understood by their parents. Watch dramas through the Internet, watch variety shows, play games, gain emotional support, satisfy resonance, find a sense of belonging, and relieve loneliness.

The above psychology leads to the behavior of young people using the Internet, such as browsing the web, watching the news, making friends, playing games, watching dramas, and chatting. Making friends and chatting through the Internet, young people can break through national boundaries and cultural restrictions through the Internet. They can get to know peers of different races, different skin colors, with the same hobbies and understand different cultures. At the same time, through communication with peers, they can gain ideological recognition. And by surpassing the limitations of space and time, the Internet has also solved the problem of young people communicating with other members of society.

\section{THE INFLUENCE OF THE INTERNET ON TEENAGERS' PSYCHOLOGY AND BEHAVIOR}

Firstly, it has some positive impact of the Internet on young people. One the one hand, the Internet helps young people learn.

The Internet provides a new way for young people to acquire knowledge. The Internet is a vast information complex. Massive and diverse information satisfies young people's thirst for knowledge. Moreover, learning in real classrooms is more inclined to teachers' theoretical explanations in textbooks, and teenagers cannot gain a more concrete understanding of knowledge. The Internet provides them with a platform for a more comprehensive understanding of classroom knowledge. And through the Internet, they can search at will and learn online courses to obtain the most cuttingedge information they need and obtain more diversified knowledge. For example, in today's Chinese society, youth sex education has become an urgent issue, and many scholars are already calling for the provision of scientific and comprehensive sex education for young people. However, due to the influence of traditional thinking, many adults still talk about sexual discoloration, thinking that young people should not be encouraged to come into contact with such knowledge. At this time, understanding scientific knowledge through the Internet to protect their health has become the choice of many young people.

On the other hand, the Internet promotes youth participation in social interactions. Youth interactions includes not only the interactions of peer groups, but also the interactions with other family members which provides a new way and path for the socialization of young people. First of all, the Internet breaks the boundaries of time, space, and identity, and gives young people new identities and roles, so that young people can meet friends of different regions, different ages, and different identities based on their hobbies through a variety of social apps. When teenagers' psychology and thoughts cannot be understood or supported in reality, the Internet helps teenagers to separate from their real identities and interpersonal relationships, prompting 
them to be more inclined to share with strangers online. On the other hand, everyone's access to the Internet enables teenagers to have more common topics with other family members. Parents can learn about the interests of today's teenagers by browsing the Internet, and have more understanding of their children's thinking. Meanwhile, the development of the Internet provides more options for communication between family members. For example, parents and children play games and watch TV dramas, which can reduce the age gap and narrow the distance between family members.

Secondly, the Internet has negative impacts on physical and mental health. Young people sitting at the table for a long-time using computers, lack of exercise, and long-term use of electronic equipment can easily lead to obesity and myopia, which will affect their health. Young people are addicted to the Internet for a long time, and communication with others is reduced in reality, leading to mental illnesses such as depression. Due to the complexity and temptation of information on the Internet, teenagers can easily lose themselves in the Internet and obtain virtual satisfaction. However, when they return to the Internet and return to reality, they will feel emptiness and frustration. Teenagers will spend more time on the Internet to gain more satisfaction which will cause Internet addiction. For example, teenagers are more likely to get praise from others in online games, and teenagers will spend more time in games in order to continue to gain this vanity and escape from real life which cause Internet addiction.

Besides, the Internet breeds juvenile crime. Violent behaviors in games and videos on the Internet can easily be imitated by young people, which in turn can cause violent attacks on other people. On September 5, 2018, three teenagers in need of money planned to rob sex workers. Later, the three teenagers confessed in court that they were imitating a BBC legal program "Crimewatch." On the other hand, some teenagers charge money for online games and live broadcast rewards, but they have no source of income themselves. They choose to steal or deceive their parents to obtain money. The Internet is a double-edged sword. While providing knowledge for young people, the Internet also contains a lot of obscene, violent, and pornographic information. Because young people have not formed correct and firm life values, this negative information will have a negative impact on the establishment of young people's correct right and wrong concepts, the moral awareness, and the healthy development of mental behavior. For example: the unhealthy information on the internet will aggravate the rebellious psychology of adolescents, and some teenagers imitate violent behavior form the internet which could cause campus bullying and campus violence.

\section{CONCLUSIONS AND SUGGESTIONS}

Although the Internet has played a positive role in facilitating the acquisition of diversified knowledge and comprehensive understanding of knowledge for young people, and the participation of young people in social interactions. On the other hand, unscientific use of the Internet can also lead to obesity, myopia, depression and internet addiction in young people. The internet will also breed juvenile delinquency. Therefore, we should start to rectify the Internet environment from the following aspects and guide young people to go online in a healthy way.

At the national level, the Ministry of Education and other six departments jointly issued the "Notice of the Ministry of Education and Other Six Departments on Jointly Carrying out Special Governance Actions on the Internet Environment for Minors" to launch a special treatment action for the online environment for minors. Carry out a clean-up campaign to clean up obscene, pornographic and bloody and violent information carried on the Internet, create a clean and tidy Internet environment for the teenagers. In the meantime, strengthen the ideological education of young people, and skillfully use the Internet to establish a correct outlook on life and values for young people.

At the same time, the native promulgated laws and regulations. The new "Criminal Law" promulgated on October 1, 1997 added computer crime clauses, stipulating the scope and time of use of the Internet by young people, and regulating the delivery of information in web pages and online games. Strengthen legal education to make young people aware of the Internet and illegal places. At the same time, the Internet is classified and the construction of the Internet's youth model is strengthened. Information cannot be completely blocked for the purpose of satisfying the mental health education of young people.

In schools, teachers should guide students to use the Internet correctly. Blindly containment measures cannot fundamentally solve teenagers' abuse of the Internet, but will stimulate their rebellion and curiosity, and have the opposite effect. Teachers can encourage young people to use mobile phones and other electronic devices to look up words, find learning materials, and expand knowledge. Parents are an indispensable part of children's education in the family. Parents should also control their children's daily online time and supervise the purpose of teenagers' use of the Internet to prevent Internet addiction. On the other hand, parents should also spend more time with their children engage in outdoor activities to divert their kid's attention from the virtual world to the reality. At the same time, for young people who already have symptoms of Internet addiction, schools and parents should actively guide them to establish correct concepts and make them 
realize that their main task as students is to learn. And also improve their sense of responsibility to their homes and society, and jointly cultivate scientific and reasonable Internet habits.

At the individual level of young people, we should improve their media literacy. When adolescents use the Internet, due to their own lack of discrimination, it is very easy to cause de-individualization. Therefore, they should enhance their ability to distinguish right from wrong and use information, and to discriminately receive information obtained from the Internet. Furthermore, they should be vigilant and guard against being deceived.

\section{REFERENCES}

[1] CNNIC. (2020). Research report on the Internet usage of minors nationwide in 2019. http://www.cnnic.net.cn/hlwfzyj/hlwxzbg/, April 1, 2021

[2] CNNIC. (2021). The 47th Statistical Report on Internet Development in China. http://www.cnnic.net.cn/hlwfzyj/hlwxzbg/qsnbg/20 2005/t20200513_71011.htm, April 1, 2021 day

[3] Yibin Shi. (2018). The influence of the Internet on the socialization of young people and educational countermeasures. China New Communications, 020(022), 203-204.

[4] Qiaolei Jiang. (2019). The psychology, risks and countermeasures of the use of short videos by teenagers. Youth Reporter, No.645(25), 17-18.

[5] YulinZan. (2005). A review of research on adolescent Internet addiction. Chinese Youth Research, 000(007), 68-71.

[6] Hongyan Zhao (2011). Research on the influence of the Internet on the socialization of adolescents. (Doctoral dissertation, Dongbei University of Finance and Economics).

[7] Shuang Wu (2013). A psychological study of cognitive dissonance in online gaming behaviors of teenagers. (Doctoral dissertation, Jilin University). 